\title{
Identification of the Enterobacteriaceae: a comparison of the Enterotube II with the API 20E
}

\author{
LJ HAYEK, GW WILLIS \\ From the Department of Microbiology, Torbay Hospital, Torquay, Devon
}

SUMMARY Two commercial methods for the identification of the Enterobacteriaceae, API 20E and Enterotube II, were compared using the results obtained with 235 cultures of fresh clinical isolates. Enterotube II was an improvement on the original Enterotube, but the availability of two differing indices, one using the Voges-Proskauer (VP) test result and one without, is criticised.

Many rapid multitest systems for the identification of the Enterobacteriaceae have appeared during the past few years. Some of these have enjoyed varying periods of popularity while others may only have been available for a short while before disappearing from the market. Two of the most successful systems, the API 20E and the Enterotube, have been available for more than ten years and, with some modifications, are still among those most widely used.

In $1976^{1}$ we compared the API $20 \mathrm{E}$ with the then available version of the Enterotube, and with the conventional methods generally used at that time in most laboratories. We found that the API results agreed almost completely $(99 \cdot 2 \%)$ with conventional methods, while the Enterotube correctly identified $85 \%$ on the first testing, with an improvement to $92 \%$ on repeat testing. In addition we found that to achieve a full identification with the Enterotube further tests had to be undertaken on $47 \%$ of the organisms, involving extra technical time and a further day's delay. We were also critical of the fact that for $7 \%$ of the organisms isolated the Enterotube relied solely on serological testing for differentiating between alternatives, while this was never the case with API.

Since then the Enterotube II has become available. This is similar in concept to the device previously described' but has been modified with four extra compartments for adonitol, arabinose, sorbitol and Voges-Proskauer (VP) tests. Results are read as before and are converted into a 5-digit numerical profile from which the identification can be found in an index. Two such indices are available, one using all the tests, while the other does not use the VP test, avoiding the necessity of waiting for the $10 \mathrm{~min}$ Accepted for publication 30 November 1983 required for any colour change to develop. The data base has now been widened to species level for most organisms, whereas the 1976 version did not go beyond genus level in the Citrobacter, Serratia and Klebsiella groups. The API 20E does not appear to have changed since we last tested it, but the data base now contains many more organisms and also many of the more unusual variants of common organisms. In addition it must be mentioned that since 1976 there have been some changes in bacterial nomenclature and new species have also been recognised. These changes have also been incorporated in the data base of both methods. We therefore felt it would now be worthwhile carrying out a study similar to our previous work to reassess the relative merits of these two systems.

\section{Material and methods}

We tested a total of 235 organisms in parallel using the API $20 \mathrm{E}$ and the Enterotube II methods according to the manufacturers' instructions. Unfortunately those organisms tested in 1976 were no longer available, but all except two of the organisms tested were isolated locally from clinical material. The remaining two organisms were a Salmonella typhi, not normally kept as one of our stock cultures, and an unusual Salmonella which had been included in a quality control scheme. Identifications were made using the indices of numerical profiles provided by the manufacturers. Both indices provided by the manufacturers of Enterotube were used.

In our previous study' we found that the API $20 \mathrm{E}$ identification agreed $100 \%$ with the conventional methods we used, while very good agreement had also been reported by other workers. ${ }^{2-5}$ We therefore felt it unnecessary to apply conventional testing 
Table 1 Cultures given the same identification by both methods on furst testing

Escherichia coli
Klebsiella pneumoniae
Klebsiella oxytoca
Enterobacter aerogenes
Enterobacter cloacae
Enterobacter agglomerans
Serratia marcescens
Serratia liquefaciens
Serratia rubidaea
Providencia stuartii
Providencia alcalifaciens
Salmonella spp
Salmonella typhi
Citrobacter freundii
Citrobacter diversus
Proteus mirabilis
Proteus vulgaris
Morganella morganii
Hafnia alvei
Enterobacter sakazakii
Shigella spp
Shigella sonnei
Edwardsiella tarda
Total

$\begin{array}{r}51 \\ 36 \\ 6 \\ 3 \\ 17 \\ 1 \\ 6 \\ 2 \\ 2 \\ 7 \\ 2 \\ 17 \\ 1 \\ 11 \\ 2 \\ 17 \\ 9 \\ 8 \\ 3 \\ 1 \\ 5 \\ 1 \\ 1 \\ 209 \\ \hline\end{array}$

methods to all organisms examined, except where required as "extras" to confirm the identification of any organisms given the same or similar names by both systems under test. Where different identifications were reached the organisms were retested by both systems and also using conventional methods.

\section{Results}

Both methods gave the same results in $209(88.9 \%)$ of the 235 organisms tested (Table 1). The remaining 26 organisms, which had been given different identifications by the two methods, were retested, and Table 2 shows how these discrepancies were resolved in 15 instances. Where the discrepancy in identification was obviously due to variation between methods of one particular test that particular test was also repeated using the relevant conven- tional method. It can be seen from Table $\mathbf{2}$ that the majority of the changed identifications were due to single false negative reactions in the Enterotube on first testing which were resolved on retesting and confirmed by a single conventional test. On two occasions there were a number of changes in the reactions given by the API system to produce the correct identification.

Table 3 lists those organisms where no agreement could be reached after retesting by both methods and no single test leading to misidentification could be identified.

The Enterotube identifications were read using both profile indices provided by the manufacturer. This led to considerable confusion, and Table 4 lists those organisms where the correct identification was only given by one of the profiles despite careful performance of all confirmatory tests recommended. In our previous study we commented on the fact that the Enterotube system required the performance of extra conventional tests before $47 \%$ of the organisms tested could be fully identified, while far fewer such tests were required by the API system. In the present study the proportion of cultures requiring such extra tests had fallen to $25 \%$ for the Enterotube, while the proportion of cultures requiring extra tests for the API system remains approximately as before at $3.4 \%$. The Enterotube system recommended serological testing alone to distinguish between alternatives in six $(2.5 \%)$ instances, being only required once by the API. This was for a most unusual organism not listed in one of the Enterotube indices or by API whose final identification by the API computer service was given as a "very doubtful identification" of Salmonella paratyphi A or Citrobacter freundii.

In this study we also compared the performance of those individual tests which are common to both systems. Results are shown in Table 5.

Table 2 Details of cultures where discrepancies in identification were resolved on retesting

\begin{tabular}{|c|c|c|c|c|}
\hline $\begin{array}{l}\text { No of } \\
\text { cultures }\end{array}$ & Enterotube & $A P I$ & $\begin{array}{l}\text { Correct } \\
\text { identification }\end{array}$ & Comments \\
\hline $\begin{array}{l}3 \\
1 \\
1 \\
2 \\
1 \\
1 \\
1\end{array}$ & $\begin{array}{l}\text { K pneumoniae } \\
E \text { aerogenes } \\
K \text { ozaenae } \\
P \text { mirabilis } \\
K \text { ozaenae } \\
P \text { mirabilis } \\
P \text { vulgaris }\end{array}$ & $\begin{array}{l}\text { K oxytoca } \\
\text { E coli } \\
K \text { pneumoniae } \\
P \text { vulgaris } \\
E \text { coli } \\
P \text { morganii } \\
P \text { morganii }\end{array}$ & $\begin{array}{l}K \text { oxytoca } \\
E \text { coli } \\
K \text { pneumoniae } \\
P \text { vulgaris } \\
E \text { coli } \\
P \text { morganii } \\
P \text { morganii }\end{array}$ & $\begin{array}{l}\text { False negative indole in Enterotube on first testing. } \\
\text { False negative indole in Enterotube on first testing. } \\
\text { False negative VP in Enterotube on first testing. } \\
\text { False negative indole in Enterotube on first testing. } \\
\text { False negative indole in Enterotube on first testing. } \\
\text { False negative indole in Enterotube on first testing. } \\
\text { False negative indole and } \mathrm{H}_{2} \mathrm{~S} \text { in Enterotube on } \\
\text { first testing. }\end{array}$ \\
\hline 1 & $S$ marcescens & S liquefaciens & $S$ marcescens & $\begin{array}{l}\text { No change in Enterotube on repeat-several } \\
\text { changes in API reactions. }\end{array}$ \\
\hline $\begin{array}{l}1 \\
2 \\
1\end{array}$ & $\begin{array}{l}\text { Cit freundii } \\
K \text { pneumoniae } \\
(1) \text { Cit freundii } \\
(2) \text { K ozoenae }\end{array}$ & $\begin{array}{l}\text { E cloacae } \\
\text { E cloacae } \\
\text { E agglomerans } \\
\text { Cit freundii }\end{array}$ & $\begin{array}{l}\text { E cloacae } \\
\text { E cloacae } \\
\text { Cit freundii }\end{array}$ & $\begin{array}{l}\text { Changes in Enterotube citrate on repeat. } \\
\text { Changes in Enterotube decarboxylases on repeat. } \\
\text { Both systems produced different identifications on } \\
\text { the first two occasions they were tested-only } \\
\text { reaching agreement the third time. }\end{array}$ \\
\hline
\end{tabular}


Table 3 Cultures where there was no agreement after retesting

\begin{tabular}{|c|c|c|c|}
\hline Enterotube & $A P I$ & $\begin{array}{l}\text { Correct } \\
\text { identification }\end{array}$ & Comments \\
\hline $\begin{array}{l}\text { Serratia rubidaea } \\
\text { Edwardsiella } \\
\text { Arizona }\end{array}$ & $\begin{array}{l}\text { K pneumoniae } \\
E \text { coli } \\
\text { Cit freundii }\end{array}$ & $\begin{array}{l}K \text { pneumoniae } \\
E \text { coli } \\
\text { Lactose-fermenting } \\
\quad \text { salmonella }\end{array}$ & $\begin{array}{l}\text { Cysteine-dependent organism. } \\
\text { Quality-control specimen. }\end{array}$ \\
\hline E cloacae & Not listed in index & Cit freundii & $\begin{array}{l}\text { API computer service contacted-suggested "doubtful } \\
\text { identification" of Cit freundii. }\end{array}$ \\
\hline Salmonella/Shigella & Cit freundii & Cit freundii & $\begin{array}{l}\text { Enterotube system required serological confirmation but no } \\
\text { alternative identification.suggested. }\end{array}$ \\
\hline $\begin{array}{l}\text { (1) E coli } \\
\text { (2) S liquefaciens }\end{array}$ & Cit freundii & Cit freundii & $\begin{array}{l}\text { Enterotube produced a different (incorrect) identification both } \\
\text { times this organism was tested. }\end{array}$ \\
\hline
\end{tabular}

Table 4 Details of organisms where the correct identification was given by one Enterotube index only

\begin{tabular}{lll}
\hline Without $V P$ & With $V P$ & Correct/API \\
\hline E cloacae & S liquefaciens & E cloacae \\
Kozaenae & Shigella/Salmonella & K ozaenae \\
E cloacae & Cit freundii & E cloacae \\
E aerogenes & Sliquefaciens & E aerogenes \\
$Y$ enterocolitica & S ozaenae & S liquefaciens \\
\hline
\end{tabular}

\section{Discussion}

On first testing the API gave the correct result in $232(98.7 \%)$ instances in all. This compares well with our previous results from 1976 and those of several other workers. ${ }^{1-5}$ On first testing the Enterotube only correctly identified $90.6 \%$ or $91.9 \%$ of the organisms examined, depending upon which profile index was used. It is true that on repeat testing the Enterotube correctly identified $97.9 \%$ of the cultures and the API $99.6 \%$, but it must be noted that such repeat testing was only carried out in an attempt to resolve obvious differences thrown up by using two methods. In normal clinical practice only one method of identification would be used on one occasion only so the accuracy of the first identification is of paramount importance, any others being of academic interest only. We were unable to confirm the results of Leighton and Little, ${ }^{4}$ who reported that the Enterotube correctly

Table 5 Comparison of tests common to both the API and the Enterotube system

\begin{tabular}{lcc}
\hline Test & $\begin{array}{c}\text { No of } \\
\text { disagreements }\end{array}$ & $\begin{array}{l}\% \\
\text { agreement }\end{array}$ \\
\hline Glucose & 0 & 100 \\
Lysine & 19 & 94 \\
Ornithine & 1 & 99.6 \\
HS & 6 & 98.2 \\
Indole & 28 & 91.6 \\
Arabinose & 9 & 97.3 \\
Sorbitol & 5 & 98.5 \\
VP & 18 & 94.6 \\
(PPA/TDA) & 9 & 97.3 \\
Urea & 22 & 93.4 \\
Citrate & 59 & 82.39 \\
\hline
\end{tabular}

identitied $95 \%$ of all organisms tested without the necessity for retesting any obviously discrepant results.

Unless a specially careful epidemiological study is being carried out it is unlikely that the misidentification of Citrobacter freundii as an Enterobacter cloacae is likely to be of very great importance to the average clinical laboratory, but as these methods are both claimed to provide accurate speciation of the Enterobacteriaceae it is unfortunate that they cannot do so without fail at first testing. The $90.6 \%$ initial success rate achieved by the Enterotube is better than the $85 \%$ we recorded eight years ago, but there still seems to be some scope for improvement. The Enterotube correctly identified all enteric pathogens, though calling the lactose-fermenting Salmonella an Arizona, while the API identified this organism as a Citrobacter. This misidentification by the API was the only significant error made by this method in our study.

Some of our previous criticism of the Enterotube concentrated on the fact that it required additional tests to be carried out on nearly half the cultures examined. This dependence on extra tests has now been greatly reduced, as has the use of serological testing as the only discriminating factor between alternatives. This is obviously due to the inclusion of four extra tests in the Enterotube battery, but it is unfortunate that the manufacturers have now complicated matters by making available two profile indices which do not quite match. One index includes all tests while the alternative index lists those profiles reached without the use of the VP test. This may save a short time at the laboratory bench but the problem is that on five occasions totally different identifications were given by the two indices for the same set of reactions. To make matters worse it was not possible to rely on either index as correct every time. On ten other occasions we noted that the same set of reactions suggested the possibility of different identifications, though when the various confirmatory tests recommended were carried out the same identification was finally reached. We feel that a careful collation of these two indices would be 
useful and add greatly to the dependability of the Enterotube.

Nord et $a^{5}$ found that the API tests had an overall 99\% agreement with conventional metheds, while similar results were reported by Smith, ${ }^{2}$ except that they reported only $91.2 \%$ correlation for the citrate and $90.4 \%$ for the urea tests. We found a considerable number of discrepancies between individual results, the worst being citrate (only $82.4 \%$ agreement) and indole $(91.6 \%)$, though it is surprising how relatively rarely some of the commonest disagreements are responsible for incorrect identification. In our previous study we found that many of the disagreements found were due to difficulties with the Enterotube decarboxylases. This particular problem seems now to have been resolved as only one misidentification could have been blamed on these reactions, though the discrepancy rate for the individual tests remained quite high, only $94 \%$ of lysine tests agreeing between the two systems. When we examined those discrepancies that were resolved on retesting (Table 4 ) it is interesting to note that most of the changes in identification for the Enterotube were due to an altered indole reaction. It is possible that this may have been due to batch variation, but it is unfortunate that there should be a lack of reproducibility of such a key reaction: The even more discrepant citrate reaction was only found to be directly responsible for one disagreement in identification.

There seems generally to have been a great improvement in the Enterotube since we last tested it in its previous form. Leighton and $\mathrm{Little}^{4}$ found that the accuracy of the two systems was almost identical, both systems correctly identifying about $95 \%$ of the specimens. We found a considerably greater difference, with the API system coming out on top once again, but we feel that there may be other factors which may outweigh some of our criticism. The Enterotube is certainly much quicker to inoculate and may at the moment also be cheaper than the API, though the latter may have a longer shelf life due to the use of dried reagents. We were disappointed with the accuracy of the Enterotube when analysing the results before retesting and hope that this may have been due to unfortunate isolated batch variations as, on retesting, there was no doubt about the firm identifications produced. We feel that despite this the Enterotube can be considered as a very useful and rapidly set up method of screening for pathogens in a busy routine laboratory. We have, however, been using the API 20E, with its larger battery of tests, for many years as our definitive method for the identification of clinically significant or unusual isolates and are of the opinion that this widely established pattern is likely to remain. We note that the Enterotube manufacturers still recommend the use of syringe and needle for the injection of the Kovacs reagent which, in these even more safety conscious days, should never be allowed in the presence of possibly dangerous pathogens. Incidentally, the syringes now in use do not seem to suffer from the use of Kovacs reagent as they did eight years ago, but that is still no excuse for advising this dangerous procedure. It is understood that the manufacturers are to devise a safer instrument for this purpose.

\section{References}

' Hayek LJ, Willis GW. A comparison of two commercial methods for the identification of the Enterobacteriaceae-API 20E and the Enterotube-with conventional methods. J Clin Pathol 1976;29: 158-61.

' Smith PB, Tomfohrde KM, Rhoden DL, Balows A. API system: a multitube micromethod for identification of Enterobacteriaceae. Appl Microbiol 1972;24:449-52.

${ }^{3}$ Brooks KA, Jens M, Sodeman TM. A clinical evaluation of the API microtube system for identification of enterobacteriaceae. Am J Med Technol 1974;40:55-61.

${ }^{4}$ Leighton PM, Little JA. Clinical comparison of the Enterotube II and API 20E systems for bacterial identification. Am J Clin Pathol 1983;79:367-70.

${ }^{5}$ Nord CE, Lindberg AA, Dählback A. Evaluation of five testkits-API, AuxoTab, Enterotube, PathoTec and R/B-for identification of Enterobacteriaceae. Med Microbiol Immunol 1974;159:211-20.

Requests for reprints to: Dr LJ Hayek, Department of Microbiology, Torbay Hospital, Torquay TQ2 7AA, England. 